\title{
Brain Tumor Detection Using MRI Images
}

\author{
Pranita Balaji Kanade ${ }^{1}$, Prof. P.P. Gumaste ${ }^{2}$ \\ Department of E\&TC Engineering, JSPMs, Jayawantrao Sawant College of Engg, Hadapsar, Pune, India ${ }^{1,2}$
}

\begin{abstract}
Most cells in the body grow and then divide in an orderly way to form new cells as they are needed to keep the body healthy and working properly. When cells lose the ability to control their growth, they divide too often and without any order. The extra cells form a mass of tissue called a tumor. Brain tumors are abnormal and uncontrolled proliferations of cells. Segmentation methods used in biomedical image processing and explores the methods useful for better segmentation. A critical appraisal of the current status of semi automated and automated methods are made for the segmentation of anatomical medical images emphasizing the advantages and disadvantages. In this project we detect the brain tumor \& classify the stages of the tumor by using testing \& training the database. Segmentation for testing purpose is done by spatial FCM used.
\end{abstract}

Keywords: Brain MR, image segmentation, learning vector quantization, self-organizing feature map, stationary wavelet transform.

\section{INTRODUCTION}

Magnetic resonance imaging (MRI) provides detailed Magnetic resonance imaging (MRI) is done for many information about brain tumor anatomy, cellular structure reasons. It is used to find problems such as tumors, and vascular supply, making it an important tool for the bleeding, injury, blood vessel diseases, or infection. MRI effective diagnosis, treatment and monitoring of the also may be done to provide more information about a disease. Magnetic resonance imaging (MRI) is a problem seen on an X-ray, ultrasound scan, or CT scan. noninvasive medical test that helps physicians diagnose Contrast material may be used during MRI to show and treat medical conditions. MRI uses a powerful abnormal tissue more clearly. A brain tumor is any magnetic field, radio frequency pulses and a computer to intracranial tumor created by abnormal and uncontrolled produce detailed pictures of organs, soft tissues, bone and cell division, normally either found in or around the brain virtually all other internal body structures. The images can itself, or spread from cancers primarily located in other then be examined on a computer monitor, transmitted organs (metastatic tumors). Primary (true) brain tumors electronically, printed or copied to a CD. MRI does not are commonly located in the posterior cranial fossa in use ionizing radiation (x-rays). Detailed MR images allow children and in the anterior two-thirds of the cerebral physicians to evaluate various parts of the body and hemispheres in adults, although they can affect any part of determine the presence of certain diseases.

Automated brain tumor detection from MRI images is one of the most challenging task in today's modern Medical imaging research. Automatic detection requires brain image segmentation, which is the process of partitioning the image into distinct regions, is one of the most important and challenging aspect of computer aided clinical diagnostic tools. Noises present in the Brain MRI images are multiplicative noises and reductions of these noises are difficult task. The minute anatomical details should not be destroyed by the process of noise removal from clinical point of view. These makes accurate segmentation of brain images a challenge. However, accurate segmentation of the MRI images is very important and crucial for the exact diagnosis by computer aided clinical tools.

A large variety of algorithms for segmentation of MRI images had been developed. Surgical planning, postsurgical assessment, abnormality detection, and many other medical application requires medical image segmentation. In spite of wide number of automatic and semi - automatic image segmentation techniques, they fail in most cases largely because of unknown and irregular noise, in-homogeneity, poor contrast and weak boundaries which are inherent to medical images. the brain.

In the United States in the year 2000, it was estimated that there were 16,500 new cases of brain tumors, which accounted for 1.4 percent of all cancers, 2.4 percent of all cancer deaths, and 20-25 percent of pediatric cancers. Ultimately, it is estimated that there are 13,000 deaths/year as a result of brain tumors. Magnetic resonance imaging (MRI) is a noninvasive medical test that helps physicians diagnose and treat medical conditions.MRI uses a powerful magnetic field, radio frequency pulses and a computer to produce detailed pictures of organs, soft tissues, bone and virtually all other internal body structures. The images can then be examined on a computer monitor, transmitted electronically, printed or copied to a CD. MRI does not use ionizing radiation ( $\mathrm{x}$ rays). Detailed MR images allow physicians to evaluate various parts of the body and determine the presence of certain diseases.

\section{Classification of Segmentation Methods}

\section{A. Image pre-processing}

Image pre-processing is the name for operations on images at the lowest level of abstraction whose aim is an improvement of the image data that suppress undesired distortions or enhances some image features important for further processing. It does not increase image information content. Its methods use the considerable 
redundancy in images. Neighbouring pixels corresponding to one object in real images have the same or similar brightness value and if a distorted pixel can be picked out from the image, it can be restored as an average value of neighbouring pixels. Image pre-processing tool, created in MatLab, realizes many brightness transformations and local pre-processing methods.

The aim of pre-processing is an improvement of the image data that suppresses undesired distortions or enhances some image features relevant for further processing and analysis task. Image pre-processing use the redundancy in images. Neighbouring pixels corresponding to one real object have the same or similar brightness value. If a distorted pixel can be picked out from the image, it can be restored as an average value of neighbouring pixels. Image pre-processing methods can be classified into categories according to the size of the pixel neighbourhood that is used for the calculation of a new pixel brightness.

\section{B. Median filters}

Median based filters have been widely used in image processing because some important image details, e.g. edges, can be retained while noise can be effectively removed by the filters. Weighted median (WM) filters are a natural extension of median filters, which exploit not only rank-order information but also spatial information of input signal. In median filtering, the neighboring pixels are ranked according to brightness (intensity) and the median value becomes the new value for the central pixel. Median filters can do an excellent job of rejecting certain types of noise, in particular, "shot" or impulse noise in which some individual pixels have extreme values. In the median filtering operation, the pixel values in the neighborhood window are ranked according to intensity, and the middle value (the median) becomes the output value for the pixel under evaluation.

The median filter are nonlinear. The median is, in a sense, a more robust "average" than the mean, as it is not affected by outliers (extreme values). Since the output pixel value is one of the neighboring values, new "unrealistic" values are not created near edges. Since edges are minimally degraded, median filters can be applied repeatedly, if necessary.

\section{The Stationary Wavelet Transform (SWT)}

Image de-noising has remained a fundamental problem in the field of image processing. With Wavelet transforms, various algorithms for de-noising in wavelet domain were introduced. Wavelets gave a superior performance in image de- noising due to its properties such as multiresolution. The problem of estimating an image that is corrupted by Additive White Gaussian Noise has been of interest for practical and theoretical reasons. Non-linear methods especially those based on wavelets have become popular due to its advantages over linear methods. An image resolution enhancement technique based on interpolation of the high frequency sub band images obtained by discrete wavelet transform (DWT) and the input image. The edges are enhanced by introducing an intermediate stage by using stationary wavelet transform (SWT). DWT is applied in order to decompose an input image into different sub bands.

Then the high frequency sub bands as well as the input image are interpolated. The estimated high frequency sub bands are being modified by using high frequency sub band obtained through SWT. Then all these sub bands are combined to generate a new high resolution image by using inverse DWT (IDWT). The Stationary wavelet transform (SWT) is a wavelet transform algorithm designed to overcome the lack of translation-invariance of the discrete wavelet transform (DWT). Translationinvariance is achieved by removing the down samplers and up samplers in the DWT and up sampling the filter coefficients by a factor of $2(j-1)$ in the $j$ th level of the algorithm. The SWT is an inherently redundant scheme as the output of each level of SWT contains the same number of samples as the input so for a decomposition of $\mathrm{N}$ levels there is a redundancy of $\mathrm{N}$ in the wavelet coefficients.

\section{Segmentation}

Segmentation is the process dividing an image into regions with similar properties such as gray level, color, texture, brightness, and contrast The role of segmentation is to subdivide the objects in an image; in case of medical image segmentation the aim is to:

- Study anatomical structure

- Identify Region of Interest i.e. locate tumor, lesion and other abnormalities

- Measure tissue volume to measure growth of tumor (also decrease in size of tumor with treatment).Help in treatment planning prior to radiation therapy; in radiation dose calculation

- Automatic segmentation of medical images is a difficult task as medical images are complex in nature and rarely have any simple linear feature. Further, the output of segmentation algorithm is affected due to partial volume effect, Intensity in homogeneity,presence of artifacts, closeness in gray level of different soft tissue

Artifacts present in MR and CT images can be divided into three categories on the basis of image processing technique needed to rectify them: (i) artifacts needing appropriate filtering technique. For example, noise artifact, susceptibility artifact and presence of nonsharp edges in the image (ii) artifact needing appropriate image restoration techniques for example motion artifacts and (iii) artifact needing specific algorithm are; partial volume, intensity inhomogeneity.

Although a number of algorithms have been proposed in the field of medical image segmentation, medical image segmentation continues to be a complex and challenging problem. Different researchers have done the classification of segmentation techniques in one or another way At present, from the medical image processing point of view we have done the classification of segmentation techniques on the basis of gray level based and textural feature based techniques. Further, we consider artificial 
intelligence as tools to optimize these basic techniques to achieve accurate segmentation results.

\section{E. Spatial FCM}

In this paper the use of spatial FCM for segmentation purpose. As one of the best image clustering methods, fuzzy local information C-means is often used for image segmentation. The effects of noise are avoided by utilizing the spatial relationship among pixels, but it often generates boundary zones for the mix pixels around the edges. This letter presents an image spatial clustering method, called fuzzy C-means with edge and local information (FELICM), which reduces the edge degradation by introducing the weights of pixels within local neighbor windows. The method can be directly applied to the image without any filter preprocessing, and the experimental results over remote sensing images show that FELICM not only effectively solves the problem of isolated and random distribution of pixels inside regions but also obtains high edge accuracies.

In order to make clustering more robust, many spatial clustering methods which can deal with original image without filtering, have been proposed. Fuzzy C-means (FCM) (BCFCM). In BCFCM, the label of a pixel is determined by both spectral features of the pixel and its mean-filtered neighbors, and a parameter $\alpha$ controls the effect of neighbors. Tarabalka et al. proposed a spectralspatial method for hyperspectral images. The homogeneous regions are obtained by combining the results of support vector machine and clustering using majority voting. To exploit the spatial information, a spatial function is defined as:

$$
h_{i j}=\sum_{k \in N B\left(x_{j}\right)} u_{i k}
$$

where $\mathrm{NB}(\mathrm{xj})$ represents a square window centered on pixel $x j$ in the spatial domain. A $3 \times 3$ window was used throughout this work. Just like the membership function, the spatial function hij represents the probability that pixel $\mathrm{xj}$ belongs to ith cluster. The spatial function of a pixel for a cluster is large if the majority of its neighborhood belongs to the same cluster. The spatial function in incorporated into membership function as follows:

$$
u_{i j}^{\prime}=\frac{u_{i j}^{p} h_{i j}^{q}}{\sum_{k=1}^{c} u_{k j}^{p} h_{k j}^{q}}
$$

Where $\mathrm{p}$ and $\mathrm{q}$ are parameters . These are control the relative importance of both functions. In a homogenous region, the spatial functions fortify the original membership, and the clustering result remains unchanged. However, for a noisy pixel, this formula reduces the weighting of a noisy cluster by the labels of its neighboring pixels. As a result, misclassified pixels from noisy regions or spurious blobs can easily be corrected.

As one of the best methods, Krinidis and Chatzis presented a robust image clustering method called fuzzy local information C-means (FLICM). FLICM is a noise insensitive method without a priori knowledge.

The clustering is dependent on both the spectral and local spatial information which cooperate by using a fuzzy factor. However, this method assumes that the label of one pixel is related to the labels of its spatial neighbors. Therefore, incorrect cluster labels may be assigned to the pixels near the edges of regions, and edges will be dislocated consequently. This letter presents an image spatial clustering method, called FCM with edge and local information (FELICM), which reduces edge degradation by introducing the weights of pixels within local neighbor windows.

\section{F. Probabilistic Nural Network (PNN)}

The Probabilistic Neural Network (PNN) algorithm represents the likelihood function of a given class as the sum of identical, isotropic Gaussians. In practice, PNN is often an excellent pattern classifier, outperforming other classifiers including backpropagation. However, it is not robust with respect to transformations of feature space, and this can lead to poor performance on certain data. We have derived an extension of PNN called Weighted PNN (WPNN) which compensates for this aw by allowing anisotropic Gaussians, i.e. Gaussians whose covariance is not a multiple of the identity matrix. The covariance is optimized using a genetic algorithm, some interesting features of which are its redundant, logarithmic encoding and large population size PNN, its lack or robustness with respect to a transformations of feature space.

Probabilistic neural networks can be used for classification problems. When an input is presented, the first layer computes distances from the input vector to the training input vectors and produces a vector whose elements indicate how close the input is to a training input. The second layer sums these contributions for each class of inputs to produce as its net output a vector of probabilities. Finally, a compete transfer function on the output of the second layer picks the maximum of these probabilities, and produces a 1 for that class and a 0 for the other classes. To correct this have proposed an extension of PNN, called WPNN, which uses anisotropic Gaussians rather than the isotropic Gaussians used by PNN.

Under the assumption that the covariance of the Gaussians is diagonal, we have described how to use a genetic algorithm to optimize the covariance for optimal performance on the training set. By the SVM we can understand only the MR image is normal or abnormal But with the help of PNN we can get the three result that weather the image is noarmal or benign or manignent. So by using PNN we can predict or classify more precisely the MR image. 
INTERNATIONAL JOURNAL OF INNOVATIVE RESEARCH IN ELECTRICAL, ELECTRONICS, INSTRUMENTATION AND CONTROL ENGINEERING Vol. 3, Issue 2, February 2015

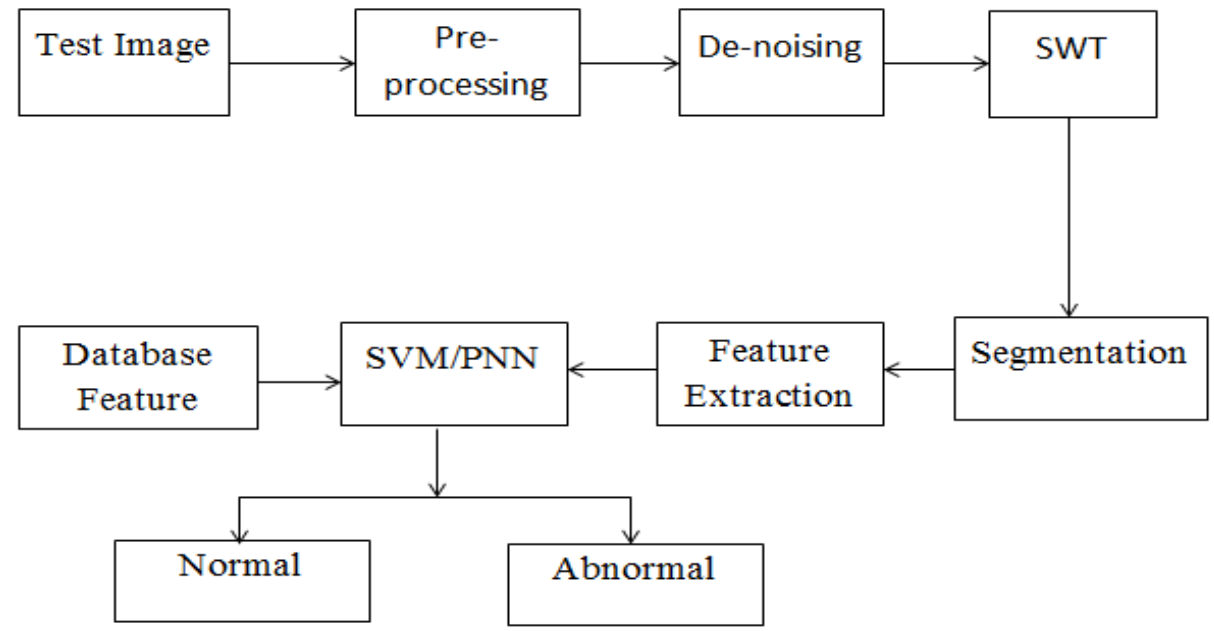

Fig.1. block diagram

\section{III.PROPOSED BLOCK DIAGRAM}

In this section we give the materials, brain MR image datasets, and methods used to perform brain MR tissue segmentation algorithm. Flow diagram of the overall algorithm that covers both training and testing processes are given in Fig.1.

The implementation details of the steps of the algorithm are discussed in the following subsections.

\section{A. Test Images}

We worked with a dataset of 15 patients that have glial brain tumors and have not gone under any surgery. The data were acquired from the medical image database in the Department of Radiology of the 29 Mayis Hospital in Ankara, Turkey. The entire database of the department was searched for brain MR images with a report of tumor.

\section{B. Pre-processing}

We normalized the intensity range of the images to [0 1$]$ range by dividing all intensity values to the maximum intensity value. To improve the signal-to-noise ratio, we applied anisotropic diffusion filter to the images as a preprocessing step. This powerful filter is defined as a diffusion process [2].

\section{De-noising \& SWT}

Median based filters and SWT technique are used for denoising the image in this paper.

\section{Segmentation}

Segmentation is the process dividing an image into regions with similar properties such as gray level, color, texture, brightness, and contrast.

\section{E. Feature Extraction}

We utilized SWT to extract features from the MR images that will be used as input to the NN. SWT is invariant to translation. SWT coefficients will not change even if the signal is shifted $[4,5]$.

\section{F. $S V M / P N N$}

In this paper the use of spatial FCM for segmentation purpose.

\section{Copyright to IJIREEICE
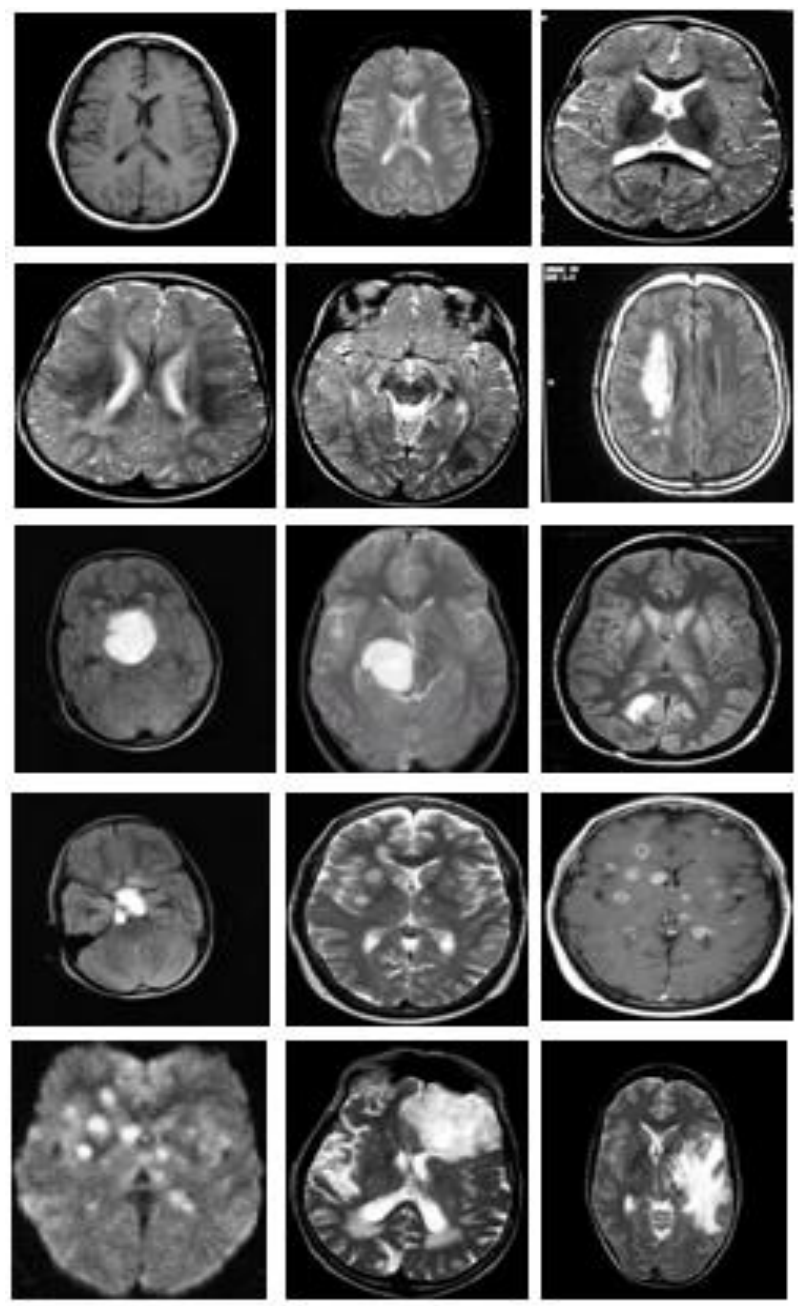

Fig.2.Different Database Test Images

\section{IV.EXPERIMENTAL RESULTS}

Following fig. 3 shows result for colour to gray \& denoised by median filter image. Out of which a colour image is Original image, Gray image is right to original image, Salt and pepper noise is below the original image, And remaining one is Median Filter image. 


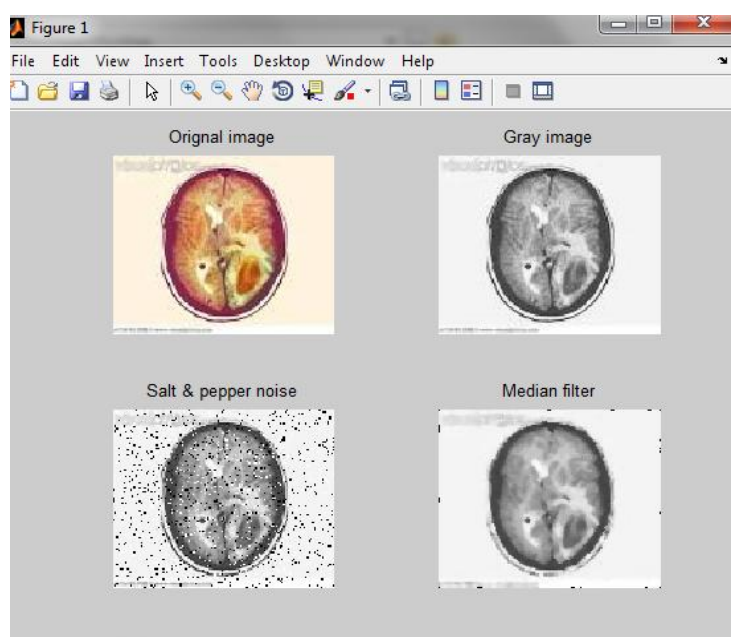

Fig.3.Result for colour to gray \& Denoised by median filter image

Following fig. 4 shows Clusturing by special FCM. Out of which upper left is cluster limage, upper right is cluster 2 image, cluster 3 is below cluster $1 \&$ cluster 4 is below cluster 2.

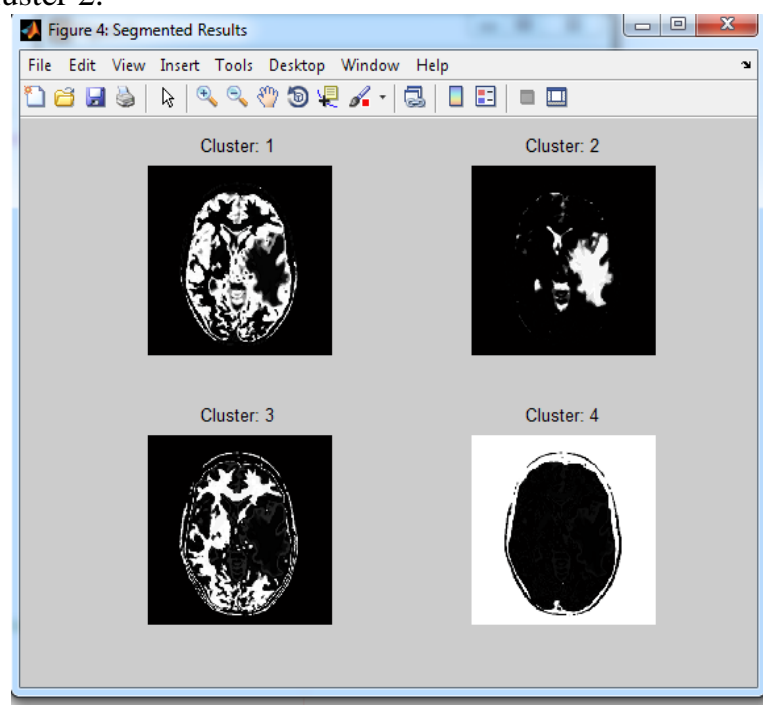

Fig.4.clustering by spatial FCM

\section{Conclusion}

The proposed algorithms for Brain Tumor Detection based on MRI Images will have higher accuracy and low error rates. The statistical analysis of the experimental results has indicated that the developed algorithm can segment brain MR images with good accuracy.

We find ways to detect these tumors at faster rate to improve survival rates. A variety of different neural network-based algorithms are also available for texturebased segmentation and classification having good accuracy. However, most of these neural network-based algorithms require extensive supervision and training and their performance depends upon the training method and data used in training. Finally, it is desired from medical image segmentation and classification algorithms that they must have the following features: a) Accuracy, b) Reliability, c) Repeatability, d) Robustness and e) Least dependency on the operator.

\section{REFERENCES}

[1] D. D. Langleben and G. M. Segall, "PET in differentiation of recurrent brain tumor from radiation injury," J. Nucl. Med., vol. 41, pp. $1861-1867,2000$

[2] A. Demirhan, İ. Güler, "Image segmentation using self-organizing maps and gray level co-occurrence matrices," J. Fac. Eng. Arch. Gazi Univ., vol. 25, no. 2, pp. 285-291, 2010.

[3] M. Kaus, S. K. Warfield, F. A. Jolesz, and R. Kikinis, "Adaptive template moderated brain tumor segmentation in MRI," in Proc. Bildverarbeitung für die Medizin, 1999, pp.102-106.

[4] M. Straka, A.L. Cruz, A. Kochl, M. Sramek, M.E. Groller, D. Fleischmann, "3D watershed transform combined with a probabilistic atlas for medical image segmentation", in Proc. MIT2003, 2003, pp. 1-8.

[5] H.-H. Chang, D. J. Valentino, G. R. Duckwiler, and A. W. Toga, "Segmentation of brain MR images using a charged fluid model," IEEE Trans. Biomed. Eng., vol. 54, no. 10, pp. 1798-1813, Oct. 2007.

[6] J. J. Corso, E. Sharon, S. Dube, S. El-Saden, U. Sinha, and A. Yuille, "Efficient multilevel brain tumor segmentation with integrated Bayesian model classification," IEEE Trans. Med. Imag., vol. 27, no. 5, pp. 629-640, May 2008.

[7] J. R. Jiménez-Alaniz, V. Medina-Bañuelos, and O. Yáñez-Suárez, "Data-driven brain MRI segmentation supported on edge confidence and a priori tissue information," IEEE Trans. Med. Imag., vol. 25, no. 1, pp. 74-83, Jan 2006.

[8] W. E. Reddick, J. O. Glass, E. N. Cook, T. D. Elkin, and R. J. Deaton, "Automated segmentation and classification of multispectral magnetic resonance images of brain using artificial neural networks," IEEE Trans. Med. Imag., vol. 16, no. 6, pp. 911918, Dec 1997.

[9] T. Song, M. M. Jamshidi, R. R. Lee, and M. Huang, "A modified probabilistic neural network for partial volume segmentation in brain MR image," IEEE Trans. Neural Netw., vol. 18, no. 5, pp. 1424-1432, Sept 2007.

[10] H. A. Vrooman, C. A. Cocosco, F. Lijn, R. Stokking, M. A. Ikram, M. W. Vernooij, M. M. B. Breteler, and W. J. Niessen, "Multispectral brain tissue segmentation using automatically trained $\mathrm{k}$ nearest-neighbor classification," NeuroImage, vol. 37, pp. 71-81, 2007.

[11] J. Alirezaie, M. E. Jernigan, and C. Nahmias, "Automatic segmentation of cerebral MR images using artificial neural networks," IEEE Trans. Nucl. Sci., vol. 45, no. 4, pp. 2174-2182, Aug 1998.

[12] S. Ahmed, K. M. Iftekharuddin, and A. Vossough, "Efficacy of texture, shape, and intensity feature fusion for posterior-fossa tumor segmentation in MRI,", IEEE Trans. Inf. Technol. Biomed., vol. 15, no. 2, pp. 206-213, Mar 2011.

[13] K. M. Iftekharuddin, J. Zheng, M. A. Islam, R. J. Ogg, "Fractalbased brain tumor detection in multimodal MRI," Applied Mathematics and Computation, vol. 207, pp. 23-41, 2009.

[14] W. Dou, S. Ruan, Y. Chen, D. Bloyet, J.-M. Constans, "A framework of fuzzy information fusion for the segmentation of brain tumor tissues on MR images," Image and Vision Computing, vol. 25, pp. 164-171, 2007.

[15] N. Zhang, S. Ruan, S. Lebonvallet, Q. Liao, Y. Zhu, "Kernel feature selection to fuse multi-spectral MRI images for brain tumor segmentation," Computer Vision and Image Understanding, vol. 115 , pp. $256-269,2011$ 